\title{
Enhanced surveillance for Salmonella Newport
}

Background and epidemiology: Between Apr. 1 and Dec. 31, 2004, Health Canada, in collaboration with provincial and territorial governments, is undertaking enhanced surveillance for Salmonella enterica serotype Newport. Physicians are urged to collect stool specimens for suspected cases of salmonellosis as part of this initiative, which resulted from several disturbing trends observed in North America. Over the past few years, there has been a rapid increase in the number of laboratoryconfirmed Salmonella Newport infections reported to the US Centers for Disease Control and Prevention (CDC). ${ }^{1}$ The spectrum of illness, which includes bloody diarrhea, tends to be quite severe ${ }^{2}$ and, according to the US National Antimicrobial Resistance Monitoring System for Enteric Bacteria, an increasing number of Salmonella Newport isolates are multidrug resistant, with the proportion of isolates resistant to 9 or more antimicrobial agents increasing from $1 \%$ in 1998 to $26 \%$ in $2001 .^{2}$ Some of these isolates also demonstrate reduced susceptibility to ceftriaxone, a thirdgeneration cephalosporin commonly used to treat invasive Salmonella infections.

In 2002 the CDC reported an outbreak of Salmonella Newport involving 47 people in 5 states, 4 of which bordered Canada. Investigation of these cases implicated exposure to raw or undercooked ground beef. The following year, Ontario veterinarians were alerted to a newly detected multidrugresistant Salmonella Newport and its rising incidence in bovine populations, primarily dairy cat$\infty$ tle. ${ }^{3}$ Three clusters of infected cattle were identified in Ontario; there were also sporadic human cases reported in Canada, at least 2 of which were related to the bovine outbreaks in Ontario. The strains isolated from these outbreaks were resistant to 9 or more antimicrobial agents and demonstrated intermediate resistance to ceftriaxone. According to the Canadian Integrated Program for Antimicrobial Resistance Surveillance and the $\mathrm{Na}$ tional Microbiology Laboratory, these extensive resistance profiles had not been observed among isolates from human sources before 2003 . $^{4}$

Direct exposure to a dairy farm, ingestion of raw milk and unpasteurized cheese, and consumption of raw or undercooked ground beef are likely risk factors for human infection. ${ }^{2}$ The purpose of Health Canada's enhanced surveillance is to increase the understanding of the burden of illness and the risk factors contributing to multidrug-resistant Salmonella Newport infections in Canada.

Clinical management: The symptoms of salmonellosis include nausea and vomiting, abdominal cramps, diarrhea, fever and headache. They can appear from 6 to 48 hours after infection with the Salmonella bacteria and usually last 1 or 2 days; however, they may be protracted in infants, elderly people and people with a weakened immune system. After 3 or 4 weeks arthritis may appear in some cases. Although antimicrobial agents are not needed in most cases of Salmonella infection, they may be life-saving in invasive infections.

Physicians are strongly encouraged to collect stool specimens from patients with suspected salmonellosis, particularly if there is a history of recent exposure to a dairy farm. For the purposes of enhanced surveillance, specimens from positive Salmonella cultures will be forwarded to provincial public health laboratories and the $\mathrm{Na}$ tional Microbiology Laboratory for serotyping and antimicrobial-resistance testing. Public health units will be notified of laboratory-confirmed cases of
Salmonella Newport infection, and health unit staff will interview cases about the illness event, risk factors (e.g., antecedent antimicrobial use) and burden of illness.

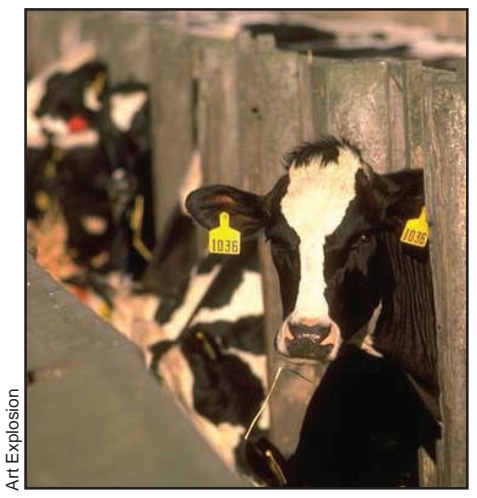

Prevention: Farming practices such as intensive farming, the tremendous movement of cattle between farms and the overuse of antimicrobial agents have probably contributed to the emergence of multidrug-resistant Salmonella Newport. Ceftiotur, an extended-spectrum cephalosporin, is commonly used therapeutically in dairy cattle, and antimicrobial agents such as tetracycline and neomycin are used prophylactically in calf milk replacers. ${ }^{2}$

Unsafe food handling and preparation are also contributing factors. Encouraging industry to implement processes such as steam pasteurization and irradiation of ground beef may reduce the risk of exposure. ${ }^{1} \mathrm{~Pa}$ tients should be reminded that meat and eggs need to be cooked to an internal temperature of $71^{\circ} \mathrm{C}$ (www.eatwelleat safe.ca/inhome/temp.htm), and utensils and surfaces used to prepare raw food should never come in contact with cooked food or foods that will be eaten raw.

Infected patients should be isolated from other household members and instructed to wash their hands. They should get time off work if working in the food industry or in health 
Practice

care facilities or if looking after people with risk factors such as immunosupression and chronic diseases such as those in nursing homes. Infected patients should have 2 consecutive negative stool samples before returning to work or normal living.

\section{Erica Weir}

Associate Medical Officer of Health

Kingston, Frontenac and Lennox

\& Addington Health Unit

Kingston, Ont.
Kathryn Doré

Acting Manager, Surveillance Section

Andrea Currie

Epidemiologist

Foodborne, Waterborne and

Zoonotic Infections Division

Centre for Infectious Disease

Prevention and Control

Population and Public Health Branch

Health Canada, Guelph, Ont.

\section{References}

1. Outbreak of multidrug-resistant Salmonella Newport - United States, January-April 2002. MMWR Morb Mortal Wkly Rep 2002;51(25):545-8.
2. Gupta A, Fontana J, Crowe C, Bolstorff B, Stout A, Van Duyne S, et al. Emergence of multidrug-resistant $\mathrm{Sal}$ monella enterica serotype Newport infections resistant to expandedspectrum cephalosporins in the United States. F Infect Dis 2003;188:1707-16.

3. Innes P. Multi-drug resistant Salmonella Newport. Toronto: Ontario Ministry of Agriculture and Food; 2003. Available: www.gov.on.ca /OMAFRA/english/livestock/vet/facts /info salm.htm (accessed 2004 June 23).

4. Canadian Integrated Program for Antimicrobial Resistance Surveillance (CIPARS). Ottawa: Health Canada 2002. Available: (English) www.hc-sc .gc.ca/pphb-dgspsp/cipars-picra/index .html (accessed 2004 June 23).

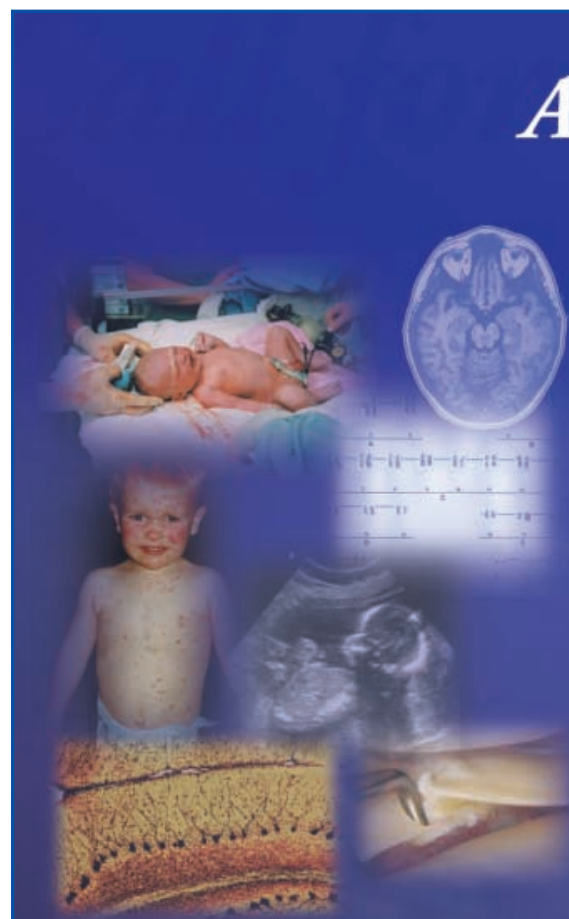

\section{A CMAJ Call for Medical Images: Clinical Vistas}

\section{Send us your interesting clinical images!}

Through scopes and scanners, on film and computer screens, with ultrasonography and microscopy, clinicians capture stunning images of illness and healing. $C M A J$ invites you to share your normally privy visual perspectives on anatomy, pathology, diagnostic procedures and therapeutic techniques. Let colleagues outside your specialty take a close look at the characteristic signs of rare conditions (Kayser-Fleischer rings in Wilson's disease) or the interior marvels of your clinical terrain (colonoscopic view of an adenomatous polyp). We're also interested in images that take a wider angle on the context of care (a recently cord-clamped newborn on a cold steel scale). If you have original, unpublished images that are beautiful or informative, rare or classic, we'd like to include them in $C M A{ }_{s}$ Clinical Vistas. Be sure to include a case summary and discussion (maximum 500 words and 4 references) and a signed consent for publication from the patient (see cmaj.ca for more instructions). Video vistas are also welcome. (See CMAJ 2004;170[13]:1931 for details.)

\section{Send your images videos or queries to:}

